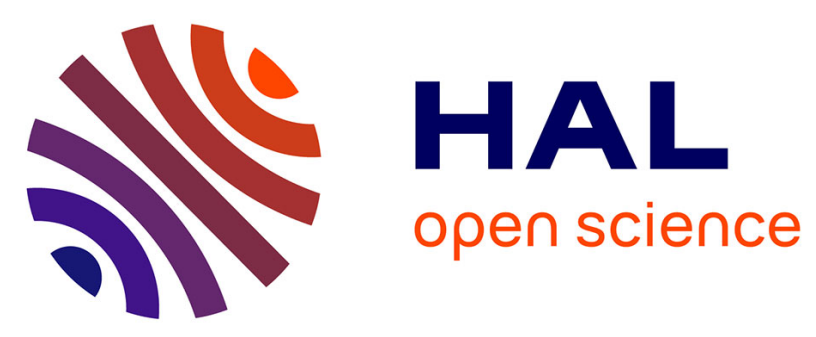

\title{
The $\mathrm{V}+\mathrm{I}$ defects in diamond: An ab initio investigation of the electronic structure, of the Raman and IR spectra, and of their possible recombination
} S Salustro, R. Dovesi, Yves Noel, C M Zicovich-Wilson, P Olivero

\section{- To cite this version:}

S Salustro, R. Dovesi, Yves Noel, C M Zicovich-Wilson, P Olivero. The V + I defects in diamond: An ab initio investigation of the electronic structure, of the Raman and IR spectra, and of their possible recombination. Journal of Chemical Physics, 2016, 145, pp.184701 - 184701. 10.1063/1.4966635 . hal-01398339

\author{
HAL Id: hal-01398339 \\ https://hal.science/hal-01398339
}

Submitted on 17 Nov 2016

HAL is a multi-disciplinary open access archive for the deposit and dissemination of scientific research documents, whether they are published or not. The documents may come from teaching and research institutions in France or abroad, or from public or private research centers.
L'archive ouverte pluridisciplinaire HAL, est destinée au dépôt et à la diffusion de documents scientifiques de niveau recherche, publiés ou non, émanant des établissements d'enseignement et de recherche français ou étrangers, des laboratoires publics ou privés. 


\title{
The V+I defects in diamond: an ab initio investigation of the electronic structure, of the Raman and IR spectra, and of their possible recombination.
}

\author{
S. Salustro and R. Dovesi \\ Dipartimento di Chimica, Università di Torino and NIS (Nanostructured \\ Interfaces and Surfaces) Centre, Via P. Giuria 5, 10125 Torino, Italy \\ Y. Nöel \\ Institut des Sciences de la Terre de Paris (UMR 7193 UPMC-CNRS), UPMC, Sorbonne Université, Paris, France \\ C. M. Zicovich-Wilson \\ Centro de Investigación en Ciencias-(IICBA), Universidad Autónoma del Estado de Morelos, \\ Av. Universidad, 1001, Col. Chamilpa, 62209 Cuernavaca (MOR), Mexico \\ P. Olivero \\ Dipartimento di Fisica, Università di Torino, Via P. Giuria 1, 10125 Torino, Italy
}

(Dated: October 17, 2016)

\begin{abstract}
The double defect in diamond, vacancy $(\mathrm{V})$ plus $\langle 100\rangle$ self split-interstitial $(\mathrm{V}+\mathrm{I})$, is investigated at the $a b$ initio quantum mechanical level, by considering the vicinal case $\mathrm{VI}_{1}$ ( $\mathrm{V}$ is one of the first neighbors of one of the two $\mathrm{C}$ atoms constituting the I defect), and the two possible "second neighbors" cases, $\mathrm{VI}_{2}^{\mathrm{D}}, \mathrm{VI}_{2}^{\mathrm{S}}$, in which a carbon atom is a first neighbor of both $\mathrm{V}$ and I. The case in which the two defects are at a larger distance is simulated by considering the two isolated defects separately $\left(\mathrm{VI}_{\infty}\right)$. A 6-21G local gaussian-type basis set and the B3LYP hybrid functional are used for most of the calculations; richer basis sets and other functionals (a global hybrid as PBE0, a range-separated hybrid as HSE06, LDA, PBE and Hartree-Fock) have also been used for comparison. With this computational approach we evaluate the energy difference between the various spin states, the location of the corresponding bands in the energy gap of pristine diamond, as well as the defect formation energy of the four defects. The path for the recombination of $\mathrm{V}$ and $\mathrm{I}$ is explored for the vicinal case, by using the Distinguished Reaction Coordinate strategy. A barrier as high as $0.75 \mathrm{eV}$ is found with B3LYP between $\mathrm{VI}_{1}$ and the perfect diamond recombined structure; when other hybrids are used, as PBE0 or HSE06, the barrier increases up to $1.01 \mathrm{eV}$ (pure DFT produces lower barriers: 0.62 and 0.67 for PBE and LDA, respectively). Such a barrier is lower than the one estimated in a very indirect way through experimental data, ranging from 1.3 to $1.7 \mathrm{eV}$. It confirms however the evidence of the extremely low recombination rate also at high temperature. The Raman (and IR) spectra of the various defects are generated, that permit to attribute unambiguously (thanks also to the graphical animation of the modes) many of the peaks observed in damaged diamond above the dominant peak of perfect bulk. For the residual non attributed peaks more complicated aggregations of defects should be explored.
\end{abstract}

\section{INTRODUCTION}

Since several decades, diamond has represented a material of high technological relevance, thanks to its extreme physical properties which make it attractive for many applications in different fields, ranging from microelectromechanical systems to heatsinks, laser windows, particle detectors, etc. $[1,2]$. In this context, the same properties that make this material technologically appealing also pose hard challenges in the micro/nanofabrication and structural functionalization. In particular, the high atomic density and strong covalent bonding of the $\mathrm{sp}^{3}$ diamond crystal determine significant issues in the control of the diffusion and recombination of structural defects.

Defects are typically created in diamond when lowfluence ion implantation is performed to either dope the material for electrical applications [3, 4] or (as explored in more recent studies) create optically active centers for applications in quantum optics $[5,6]$. In this process, the formation of both interstitial and vacancy point defects in lightly-damaged diamond has been widely observed in previous works, thanks to the unequivocal attribution of characteristic optical absorption and photoluminescence features, i.e. the GR1 $(\lambda=742 \mathrm{~nm})$ and ND1 $(\lambda=394$ $\mathrm{nm})$ lines corresponding respectively to the neutral and negatively charged vacancy, and the $3 \mathrm{H}$ emission $(\lambda=503$ $\mathrm{nm}$ ) for the interstitial.[7-9]

While in the case of electrical functionalization the creation of Frenkel pairs in the diamond structure is generally regarded as an undesiderable side-effect of the implantation of doping elements[10], in quantum-optical applications the creation of vacancies and/or interstitials can play a key role in the creation of the desired optically active complexes[11, 12]. In any case, once the desired complexes are activated, it is highly suitable to efficiently remove all residual defects from the material. To this scope, thermal annealing is typically performed at the highest possible temperature, i.e. close to the Debye temperature of diamond $(\sim 1800 \mathrm{~K})$, above which 
the diamond structure starts graphitizing. Regardless of these efforts, it is well known that residual damage in the diamond structure is extremely hard to remove, and multiple works have reported on a partial degradation of both the optical[13, 14] and electronic[10, 15] properties of diamond devices subjected to ion implantation in their fabrication steps, even after prolonged high-temperature annealing. Similar considerations can be made in the case of high-fluence implantation of diamond, which is typically aimed at the creation of localized graphitic phases in the crystal for the fabrication of electrically conductive structures[16] or for the implementation of the socalled "lift-off" fabrication process[17]: residual structural damage is generally detected in the regions damaged at low densities (i.e. well below the graphitization threshold) even after thermal processing of the final structures. Also in this case, such residual damage is undesiderable for all technological applications requiring high-quality diamond substrates[18].

The recombination of residual Frenkel defects in diamond after ion implantation and subsequent annealing has been extensively investigated in a series of experimental works based on both ion[19] and electron[20] irradiation, and interpreted with articulated models based on reaction-rate theory, which allowed for a phenomenological description of the activation energies for both the diffusion and recombination of self-interstitials and foreign interstitials with vacancies $[21,22]$. It was found that, when a vacancy and a self-interstitial diffuse within the same so-called "catch-volume" [21], a specific energy barrier needs to be overcome in order for recombination to occur. In particular, studies of the evolution of the vacancy-related EPR and luminescence signals at different annealing steps of $\mathrm{MeV}$-electron-implanted samples led to estimations of this recombination barrier varying between $1.68 \pm 0.15$ $\mathrm{eV}[8], 1.6(2) \mathrm{eV}[23]$ and $\sim 1.3 \mathrm{eV}[20]$.

It is worth remarking that, although the main subject of the present work is the investigation of the direct vacancy-interstitial recombination mechanism, alternative pathways involving more complex defects can either facilitate this process or be competitive with it, thus effectively modifying the recombination efficiency during thermal processes. For example, it is well known that the common nitrogen impurity can play a significant role in vacancy trapping, with the single substitutional impurities being $\approx 8$ times more effective in this process with respect to the $\mathrm{N}=\mathrm{N}$ complexes (usually referred as A-centres).[24] This is demonstrated by the experimental observation that the vacancy recombination process is less efficient in type IaA diamonds (which are rich in A-centres), compared to high-purity type IIa diamonds. [7, 25] As another example, the aggregation of two vacancies into a stable divacancy cluster is a process which is competitive with the interstitial/vacancy recombination process discussed here.[26]

From the simulation point of view, one of the crucial issues is the appropriate description of the defect states in the gap of the semiconductor, and the various possible spin couplings. These points have been discussed about 60 years ago in a seminal paper by Coulson[27], using an extremely simple model. When ab initio techniques became feasible, both the cluster and the supercell approaches have been employed[28-30], in most of the cases in conjuction with the simplest formulations of DFT (i.e. LDA or PBE). These approaches are however known to poorly describe the exchange interaction of unpaired electrons (with a consequent low or null localization of these electrons) and to largely underestimate the band gaps in which the defect states are located. This issue is particularly important in the present case, as overall six uncoupled electrons are involved: four on the atoms around the vacancy $[31,32]$, and two on the atoms forming the $\langle 100\rangle$ interstitial [33].

In the present paper, the $a b$ initio supercell scheme of the CRYSTAL14 code[34] is considered to the study of the vacancy plus interstitial $(\mathrm{V}+\mathrm{I})$ couple of defects in diamond, by adopting a local gaussian-type basis set and various "hybrid" functionals, that contain a fraction of exact exchange. The reciprocal perturbation of the two defects as a function of their distance and orientation, the path for the possible recombination when they are at their first neighbors, the nature of the corresponding transition state (TS) and the height of the energy barrier are investigated. The paper is structured as follows. Subsection II A illustrates the four models adopted here for the vacancy-interstitial $(\mathrm{V}+\mathrm{I})$ defect (relative orientation and distance). In Subsection II B, we discuss the algorithm adopted for determining the path for the recombination of first-neighboring vacancy and interstitial defects, as well as the strategy for the identification of the TS. Section III is organized in four parts. In subsection III A the band structure of the various defects is compared to the one of perfect diamond. Subsection III B refers to the analysis of the electronic states for the 4 types of defect couples and of the relative energy of the various spin states. In Subsection III C, the transition state is analyzed. In Subsection III D, the vibrational features of the defects are reported and analyzed in comparison with experimental findings. Finally, the conclusions are drawn in Section IV. In the Supplementary Material (SM) the computational setup (the adopted basis sets, functionals, computational tolerances, the way the vibrational frequencies and the Raman intensities are obtained) is reported, as well as the Mulliken analysis of the charge and spin distribution (to be compared with the periodic Hirshfeld-I data shown in the following) and the IR spectra.

\section{METHODS AND MODELS}

\section{A. Models for the Vacancy plus interstitial double defect}

A supercell approach is used to simulate different concentrations of defects. Supercells containing $64\left(\mathrm{~S}_{64}\right)$ 
and $128\left(\mathrm{~S}_{128}\right)$ atoms have been considered. As regards the relative position of the two defects (the vacancy $\mathrm{V}$ and the interstitial I), four cases have been considered. Firstly, the two defects are first neighbors (indicated as $\mathrm{VI}_{1}$ in Figure 1, on the right). Secondly, the secondneighbor defects are separated by a carbon atom: the two possible cases are indicated as $\mathrm{VI}_{2}^{\mathrm{D}}$ (Figure 1, left), $\mathrm{VI}_{2}^{\mathrm{S}}$ (Figure 1, center), where superscripts D and $\mathrm{S}$ indicate the presence of a double or a single bond, respectively. Finally, the case in which the two defects are separated by a larger distance is simulated by considering the two isolated defects separately $\left(\mathrm{VI}_{\infty}\right)$. The distances reported in Figure 1 (right) refer to $\mathrm{VI}_{\infty}$, as obtained in previous studies of the two separated defects [31-33].

In the $\mathrm{V}+\mathrm{I}$ cell, six uncoupled electrons (four on the vacancy, two on the interstitial) can combine to give states with four possible $\mathrm{S}_{z}$ spin values, i.e.: $3,2,1$ and 0 . The different spin states have been generated starting from the highest spin state $\mathrm{S}_{z}=3$, easily obtained by using the SPINLOCK and ATOMSPIN options ( $c f r$. CRYSTAL manual [35]); lower spin states down to $\mathrm{S}_{z}=0$ have been obtained by the SPINEDIT option that "reverses" the spin on specific atoms. It is worth underlining that only the total spin of the unit cell (i.e. not the one on each atom) is imposed with the SPINLOCK option.

\section{B. Searching for the Transition State}

Three different optimization strategies have been considered. Stable species characterized as minima of the Potential Energy Surface (PES) were fully optimized under symmetry constraints with the default code conditions. [35] The $\mathrm{S}_{z}$ values of the different considered states have been kept fixed along the optimizations by imposing them in the SCF parts of calculations, as detailed in the previous Section. While it is never
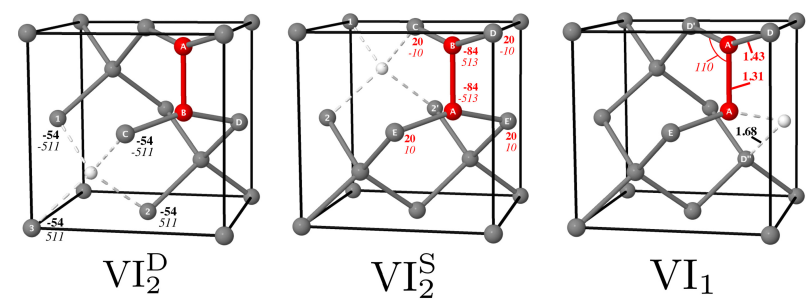

FIG. 1: (color online) Schematic representation (before geometry optimization) of the $\mathrm{VI}_{2}^{\mathrm{D}}, \mathrm{VI}_{2}^{\mathrm{S}}$ and $\mathrm{VI}_{1}$ defects. The atoms that form the interstitial defect are in red, while the vacancy is reported in light grey. Numbers on the figure to the left (center) are the Hirshfeld electron net charges ( $q$, bold) and spin momenta ( $\mu$, italic) as resulting from the calculation of the vacancy (interstitial) isolated defect; numbers on the right figure are the corresponding distances (in $\AA$, bold) and angles (in degrees, italic). $q$ and $\mu$ are expressed in millielectrons. Vacancy numbers in black, interstitial numbers in red. warranted that the most stable of such structures among those of similar structural arrangement and different spin state is the "true" ground state, we shall assume so in the following analysis of the results.

Minimal energy paths connecting stable structures along the PES were characterized by means of the so-called Distinguished Reaction Coordinate (DRC) scan.[36, 37] Finally, the highest energy points along such paths were computed with a suitable TS optimization technique [37], so as to accurately estimate the energy barrier of the process.

The DRC method requires the definition of one geometrical parameter that mainly controls the process and can be used to scan the reaction path. Such an exploration is performed by keeping fixed the chosen coordinate at a set of values within a given range while relaxing the remaining degrees of freedom by restricted energy minimization. In most cases, the processes of interest involves bond cleavage or formation. Accordingly, the DRC scan is most efficiently performed in terms of valence internal coordinates, namely bond lengths, angles and torsionals. Such a kind of geometrical parameters are defined in CRYSTAL14 for optimizations under the so-called redundant scheme. [35, 37-39]

In the original proposal for molecules [38] a redundant valence coordinate system is built up by first searching and classifying every couple of centers that are separated by a distance smaller than the sum of the respective (covalent or ionic) bonding radii. This defines a given connectivity for the structure. When unconnected structural fragments still appear, additional pairs of arbitrarily chosen atoms of each fragment are considered for connection. This feature warrants the required full structural connectivity condition.[38] Once such a classification is done, the valence internal coordinates that only involve connected centers are then automatically included in a set to define a coordinate system. This approach is redundant because in general it involves a much larger number of coordinates than the allowed degrees of freedom. Nonetheless, apart from accidental linear dependences, it provides a basis set that spans the full space of nuclear displacements.

The previous scheme has been generalized for periodic systems and implemented in CRYSTAL14.[37] It is worth noting that in structures displaying a large number of connectivity closed loops (as it is the case for most periodic systems) the number of redundant valence coordinates generated by the previous automatic prescription may become extremely large. This could make the strategy unfeasible for structures with a significant number of atoms per unit cell.

In the present work, a modification of the previous redundant valence scheme has been adopted. The redundant coordinate system is partially made of a restricted set of valence parameters, automatically generated starting from a few "bond lengths" between those atoms that are expected to be the main participants in the reaction path. To ensure that the 
entire space of nuclear displacements is spanned by the geometrical parameters under consideration, a full system of crystallographic-like coordinates is redundantly included in the coordinate system. The scheme, using a machinery similar to that considered in the full valence redundant scheme, [37, 38] allows to efficiently perform the required DRC scans. At the same time, it prevents the previously mentioned explosion of the size of vectors and matrices usually occurring when a full valence coordinate system is considered.

Starting in most cases from the highest energy point of the scan, a TS optimization[37] is subsequently performed so as to accurately determine the energy barrier of the process. The recommended choice for this kind of optimizations is to numerically compute second derivatives so as to construct the exact Hessian matrix in the first optimization point. This is performed in a way similar to what reported in Eq. S1, but it only involves totally symmetric atomic and lattice vector displacements. For numerical second derivatives the forward-point formula has been considered. The Hessian matrix is then updated at each optimization step by means of the Bofill's prescription.[40] The TS optimization scheme iterates up to fulfill the same convergence criteria as the minimization one. To ensure that the search remains within the TS region, the maximum allowed coordinate change between steps is much more severely constrained than in minimizations. Here, a limiting value for such displacements of 0.05 in generalized units has been considered (see the MAXTRADIUS keyword in the CRYSTAL code manual[35]).

\section{RESULTS}

\section{A. Band structures}

The band structure of the three defects are shown in Figure 2, where also the perfect diamond structure is reported for comparison. The band structure of $\mathrm{VI}_{1}$ is very close to the one of perfect diamond, without defect energy levels in the gap. On the contrary in $\mathrm{VI}_{2}^{\mathrm{D}}$ and $\mathrm{VI}_{2}^{\mathrm{S}}$ many defect states nearly fill the original gap of diamond, confirming their lower stability with respect to $\mathrm{VI}_{1}$.

\section{B. Geometry and charge and spin distribution}

Our analysis starts from the case in which the two defects are separated by a large distance, i.e. $\mathrm{VI}_{\infty}$, so that they do not interact; energy data are shown in the lowest part of Table I. In the isolated vacancy[32], four uncoupled electrons on the four first neighbor carbon atoms generate three spin states, characterized by $\mathrm{S}_{z}=2,1$ and 0 , the former being much less stable than the two latter ones. The $\mathrm{S}_{z}=0$ spin state is more stable than $\mathrm{S}_{z}=1$ by $0.14 \mathrm{eV}$. Also in the $\langle 100\rangle$

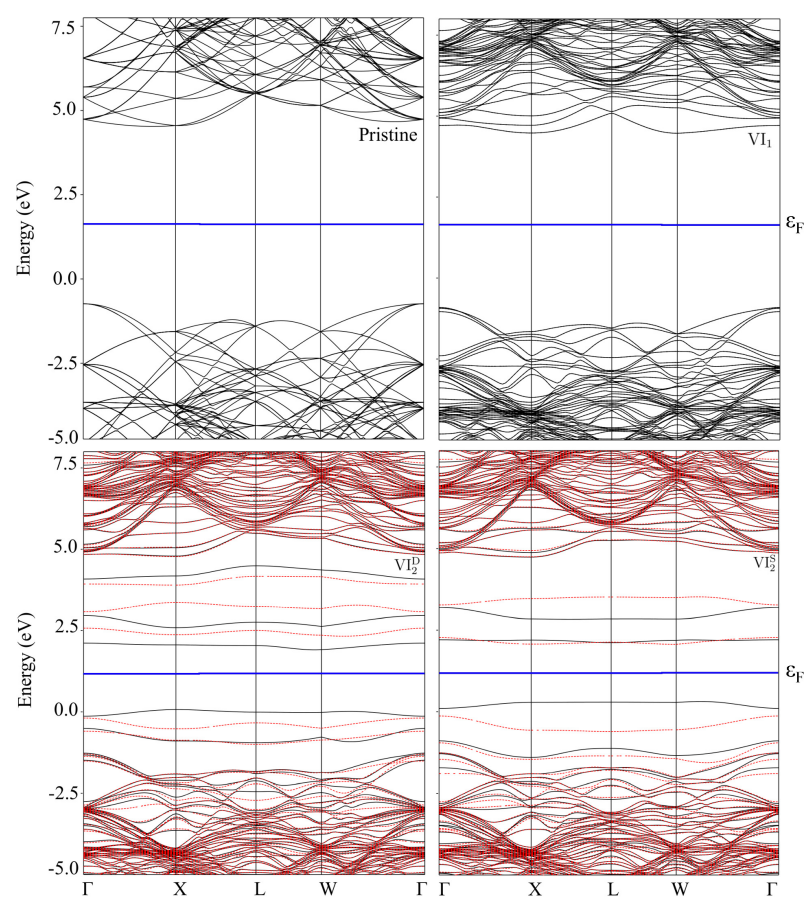

FIG. 2: (color online) Pristine, $\mathrm{VI}_{1}, \mathrm{VI}_{2}^{\mathrm{D}}$ and $\mathrm{VI}_{2}^{\mathrm{S}}$ B3LYP/621 band structures for the $\mathrm{S}_{z}=0$ spin state and $\mathrm{S}_{128}$ supercell. Continuous black and dashed red lines refer to $\alpha$ and $\beta$ electron energy levels, respectively. Continuous blue line marks the position of the Fermi energy level.

self split-interstitial[33], the $\mathrm{S}_{z}=0$ state is more stable than the $\mathrm{S}_{z}=1$ one; in this case the energy difference is however five times smaller $(0.03 \mathrm{eV})$. In summary, the overall system has a singlet ground state that is more stable than the two triplet states, corresponding to the excitation of the vacancy $\left(\mathrm{S}_{z}(\mathrm{~V})=1\right)$ or of the interstitial $\left(\mathrm{S}_{z}(\mathrm{I})=1\right)$; the quintuplet originated from the triplet state of the two defects is only $0.18 \mathrm{eV}$ less stable than the singlet. The defect formation energy is stable with respect to the defect concentration: the difference between the results obtained from $\mathrm{S}_{64}$ and $\mathrm{S}_{128}$ is of the order of $1 \%$ (see Table I). The $\mathrm{S}_{z}=3$ high spin state is $1.4 \mathrm{eV}$ less stable than the $\mathrm{S}_{z}=0$ state.

As regards charge and spin distribution, Hirshfeld-I[41] and Mulliken population analyses have been performed. The net atomic charges $q$ and spin momenta $\mu$ obtained with the former are reported in Figure 1 (left and centre, whereas some distances are shown on the right), whereas the Mulliken data are shown in Table S1 of the SM file. The $\mu$ values for the Hirshfeld-I $S_{z}=0$ state are in both cases about $0.5|e|$ (they are close to the one in the Mulliken case), whereas $q$ is only slightly negative (-0.05 $|e|$ and $-0.08|e|$, respectively). The $\mathrm{C}-\mathrm{C}$ distance in the interstitial (A-A' in Figure 1) is much shorter than in perfect diamond $(1.31 \AA$ instead of $1.56 \AA)$; the four neighbors of the vacancy relax outwards by $0.12 \AA$.

We can now consider the cases in which the two defects 
TABLE I: Formation energy $\left(\mathrm{E}_{f}\right)$ and relative stability $(\Delta \mathrm{E})$ of various $\mathrm{S}_{z}$ spin states for the vacancy plus interstitial (VI) defects at two concentrations. T, V and I are the values of $\mathrm{S}_{z}$ for the total cell and the $\mathrm{V}$ and I sites, respectively. The 1 , 2 and $\infty$ subscripts indicate that the two defects are first or second neighbors, or at infinite distance. Superscripts $S$ and $D$ label the two different second neighbors cases, and indicate that a single or a double bond is formed. Energies in $\mathrm{eV}$.

\begin{tabular}{cccccccc}
\hline \hline \multirow{2}{*}{ System } & \multicolumn{3}{c}{$\mathrm{S}_{z}$} & \multicolumn{3}{c}{$\mathrm{S}_{64}$} & \multicolumn{2}{c}{$\mathrm{S}_{128}$} \\
\hline \multirow{3}{*}{$\mathrm{VI}_{2}^{\mathrm{D}}$} & $\mathrm{T}$ & $\mathrm{V}$ & $\mathrm{I}$ & $\mathrm{E}_{f}$ & $\Delta \mathrm{E}$ & $\mathrm{E}_{f}$ & $\Delta \mathrm{E}$ \\
& 2 & 2 & 1 & 19.16 & 3.23 & 19.46 & 3.25 \\
& 0 & 0 & 0 & 15.93 & - & 16.21 & - \\
\hline \multirow{4}{*}{$\mathrm{VI}_{2}^{\mathrm{S}}$} & 3 & 2 & 1 & 18.89 & 2.71 & 19.31 & 2.73 \\
& 2 & 1 & 1 & 16.78 & 0.60 & 17.17 & 0.59 \\
& 1 & 0 & 1 & 16.19 & 0.01 & 16.58 & $0.00(3)$ \\
& 0 & 0 & 0 & 16.18 & - & 16.58 & - \\
\hline \multirow{3}{*}{$\mathrm{VI}_{1}$} & 3 & 2 & 1 & 18.95 & 11.89 & 19.24 & 11.80 \\
& 2 & 1 & 1 & 15.08 & 8.02 & 15.90 & 8.46 \\
& 0 & 0 & 0 & 7.06 & - & 7.47 & - \\
\hline \multirow{3}{*}{$\mathrm{VI}_{\infty}$} & 3 & 2 & 1 & 19.54 & 1.44 & 19.75 & 1.43 \\
& 2 & 1 & 1 & 18.28 & 0.18 & 18.49 & 0.17 \\
& 0 & 0 & 0 & 18.10 & - & 18.32 & - \\
\hline \hline
\end{tabular}

are approaching. When defects are separated by two carbon atoms $\left(\mathrm{VI}_{3}\right)$, their interaction is negligible (data not reported here). On the contrary, for the $\mathrm{VI}_{2}$ and $\mathrm{VI}_{1}$ cases (the two defects are either second or first neighbors), the reciprocal perturbation becomes very significant. Figure 1 shows the relative position for the three cases under consideration, namely $\mathrm{VI}_{2}^{\mathrm{D}}, \mathrm{VI}_{2}^{\mathrm{S}}$ and $\mathrm{VI}_{1}$ before the geometry optimization (the atomic positions of the first neighbors of the two defects are the ones resulting from the optimization of the two separated defects). All the structural figures are also shown in the web-site at Reference 42, where they can be rotated for a better evidence of the relative position of atoms. In the $\mathrm{VI}_{2}^{\mathrm{D}}$ case, the reciprocal orientation of the two defects is such that a double bond can be formed, as shown in Figure 3, referring to the fully optimized geometry ( $\mathrm{S}_{64}$ supercell). The six involved carbon atoms are not fully co-planar (this would represent the ideal situation for a double bond between two three-coordinated carbon atoms); however only one of them is out of plane by a significant amount (i.e. $52^{\circ}$ ). The optimized geometry shows two rather short bonds at about $1.32 \AA$ (A-B and B-C; note that $\mathrm{A}, \mathrm{B}, \mathrm{C}$ are three-coordinated), to be compared to 1.56 $\AA$ in perfect diamond and $1.30 \AA$ for the isolated I defect[33]. A-B (see Figure 3) is so short due to the strong local compression caused by the insertion of an additional atom in the I defect; on the contrary, B-C shortens due to the double bond, as confirmed by the Mulliken Bond Population data: the B-C population is $0.52|e|$, to be compared with $0.39|e|$ obtained for

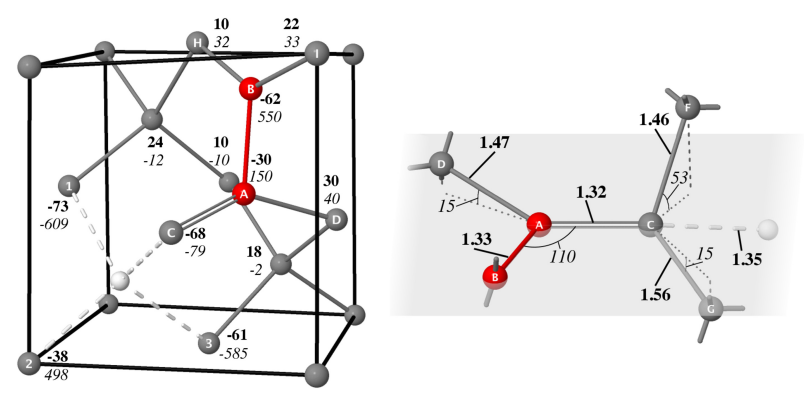

FIG. 3: (color online) To the left, a schematic representation of the $\mathrm{VI}_{2}^{\mathrm{D}}$ defect; reported numbers are the Hirshfeld electron net charges $(q$, bold) and spin momenta ( $\mu$, italic) in millielectrons. Atoms A and B (in red) form the $\langle 100\rangle$ self split-interstitial. A and $\mathrm{C}$ are involved in a double bond and are three fold coordinated, with B, D and F, G first neighbors. Also B is threefold coordinated. Atoms 1, 2, 3, C are the first neighbors of the vacancy. Bond lengths and angles with respect to the grey plane defined by atoms $\mathrm{A}, \mathrm{B}$ and $\mathrm{C}$ are reported to the right.

$\mathrm{VI}_{\infty}$.[33] Note that the $\mathrm{C}-\mathrm{C}$ bond population in pure diamond is $0.30|e|$; in the $\mathrm{C}_{6} \mathrm{H}_{12}$ molecule, with the six carbon atoms lying on the same plane, it is $0.62|e|$ when the same basis set and functional are used. The $\mu$ value depicts a quite clear electronic structure: it is around 0.5-0.6 $|e|$ (positive or negative to give the $\mathrm{S}_{z}=0$ state) on atoms 1, 2, 3 (first neighbors of the vacancy) and on $\mathrm{B}$ (one of the two atoms involved in the interstitial defect); on $\mathrm{A}$ and $\mathrm{C} \mu$ is much smaller $(+0.150|e|$ and $-0.079|e|$, respectively), although not null because the double bond is not perfect as the six involved atoms are not fully coplanar. The energy gain due to the

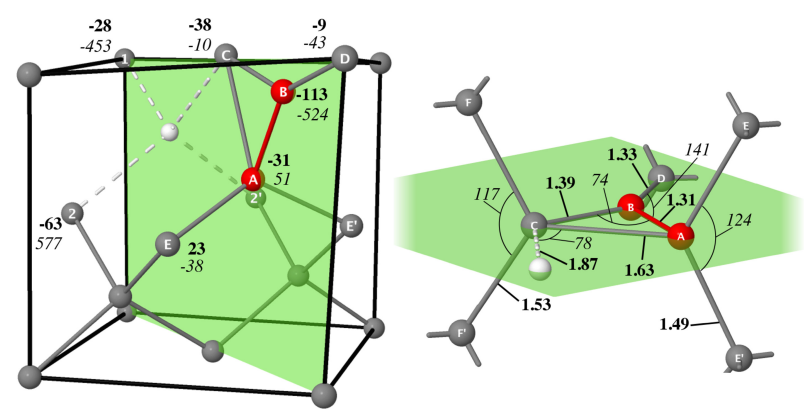

FIG. 4: (color online) Equilibrium geometry of $\mathrm{VI}_{2}^{\mathrm{S}}$. The equivalent of the conventional diamond cell has been represented to the left with Hirshfeld electron net charges ( $q$, bold) and spin momenta ( $\mu$, italic); $q$ and $\mu$ in millielectrons; a zoom on the defect is shown to the right with distances (in $\AA$, bold) and angles (in degrees, italic). Red and white atoms represent the $\langle 100\rangle$ self split interstitial and the vacancy, respectively. The symmetry plane (translucent green) contains atoms A, B, $\mathrm{C}, \mathrm{D}$ and 1 ; only non equivalent distances are reported. Bond length in vacancy is evaluated with respect to the centroid of the first nearest neighbors (C, 1, 2 and 2'). 
formation of the double bond is in part counterbalanced by the strong deformation around the defect, so that the formation energy lowers by only $2 \mathrm{eV}$ (from $18 \mathrm{eV}$ to 16 $\mathrm{eV}$ ) with respect to $\mathrm{VI}_{\infty}$. The energy difference between the triplet(V)-triplet(I) and singlet(V)-singlet(I) states is small $(0.21 \mathrm{eV})$, and not far from the one of $\mathrm{VI}_{\infty}$, thus confirming that the two states remain competitive, and can both be populated at high temperature. The band structure (see figure 2) shows many bands in the gap of perfect diamond, due to defect states. Note that the $\alpha$ and $\beta$ defect energy levels exhibit some splitting.

Also in the $\mathrm{VI}_{2}^{\mathrm{S}}$ case, two of the six three-fold coordinated atoms of the two isolated defects can exploit their short mutual distance and become fourfold coordinated: atom $\mathrm{C}$, with neighbors at $1.29,1.53,1.53$ and $1.63 \AA$ (see Figure 4, right) and atom A with neighbors at 1.31, $1.49,1.49$ and $1.63 \AA$. The spin momenta $\mu$ on $1,2,2$ ', and $\mathrm{B}$ are similar to the ones observed for $\mathrm{VI}_{2}^{\mathrm{D}}$. The formation energy is slightly larger than the $\mathrm{VI}_{2}^{\mathrm{D}}$ one: 16.18 vs $15.93 \mathrm{eV}$ for the $\mathrm{S}_{64}$ cell (it is $0.3-0.4 \mathrm{eV}$ larger for $\left.\mathrm{S}_{128}\right)$. The singlet state is only slightly more stable than the triplet state obtained by reversing the spin on atom B.

The vicinal $\mathrm{VI}_{1}$ defect is much more stable than all previously described $\mathrm{V}+\mathrm{I}$ defects, as in this case all the involved atoms are four-fold coordinated, although distances and angles are quite far from the perfect situation of diamond: one bond is shorter $(1.40 \AA)$, and one longer $(1.70 \AA)$ than in the perfect material $(1.56$ $\AA$ ), whereas the other two bonds are nearly the same as in perfect bulk $(1.57 \AA)$. Due to symmetry, the situation around A and A' is the same. The formation energy is 7-7.5 eV only, i.e. about $10 \mathrm{eV}$ less than for the three other cases. The ground state is a closed shell (see Table S1 in SM file) and the band structure is much closer to the one of perfect diamond: the gap is "clean", without

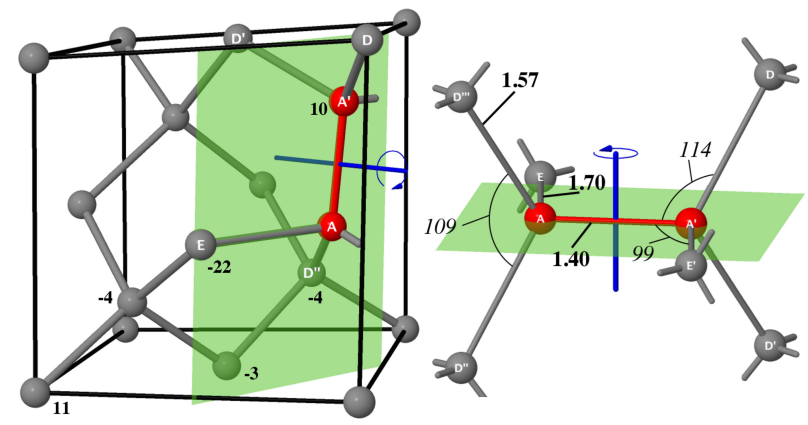

FIG. 5: (color online) Distances (in $\AA$ ) and angles (in degrees) of the $\mathrm{VI}_{1}$ equilibrium structure (right). Numbers on the left figure are the Hirshfeld charges (in millielectrons). The solution is not spin polarized. Interstitial atoms in red. The same label is used for symmetry related atoms (A and A', and the four $\mathrm{B}$ atoms). The mirror plane and the $\mathrm{C}_{2}$ rotation axis are shown in green and blue, respectively (their product, the inversion operator, is not shown). defect states inside, as shown in Figure 2.

\section{The possible recombination of the I and V defects}

The recombination of interstitial and vacancy defects is here studied for the $\mathrm{VI}_{1}$ case (see Figure 1). To model the structures appearing along the recombination path, a $\mathrm{S}_{64}$ supercell exhibiting a $C 2 / m$ space group symmetry has been considered. A schematic view of the atoms involved in the process is shown in Figures 5 and 6 . The recombination path has been scanned according to the DRC scheme described in Section IIB. The E-A and its symmetry-equivalent atomic distances have been considered as leading parameters of the process and have been simultaneously scanned exploiting their one-to-one correspondence. The animation of the atoms along the path, and the energy profile are shown at the web-site reported in Reference 42 . For such bond lengths, ten evenly spaced values in the $1.70-2.52 \AA$ range were taken into account for scanning. Calculations were performed at the B3LYP/6-21G computational level and looking at closed shell solutions only, as both the starting $\left(\mathrm{VI}_{1}\right)$ and final (pure diamond) ground states are closed shells. The obtained energy profile for the process is shown in Fig. 7. The corresponding geometries are shown in a one-to-one correspondence with the energies in the animation at the web-site reported in Reference 42 .

Starting from the structure of maximum energy along the scan, which is the fifth one according to the energy profile shown in Figure 7, a TS optimization was performed that fastly converged in 16 iterations. The vibrational analysis of the obtained structure presents a single imaginary frequency at $910 i \mathrm{~cm}^{-1}$, confirming its TS nature. The rather abrupt changes featured by the
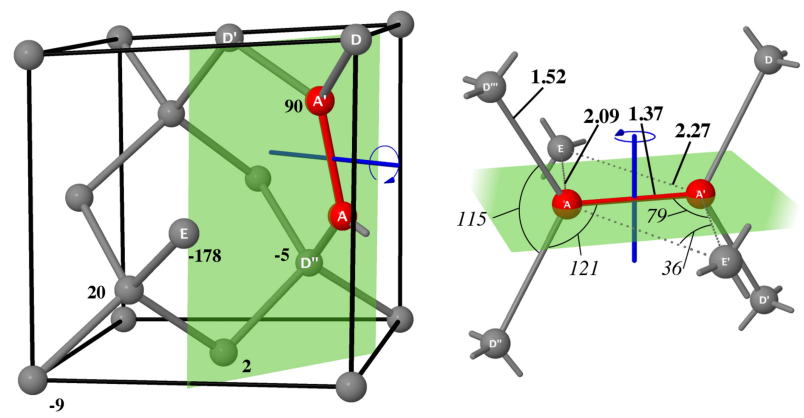

FIG. 6: (color online) The transition state between the $\mathrm{VI}_{1}$ and the perfect diamond structures. Distances (in $\AA$ ) and angles (in degrees) are reported to the right, Hirshfeld charges (in millielectrons) to the left. Interstitial atoms in red. The same label is used for symmetry related atoms (A and A', and the four B atoms). The mirror plane and the $C_{2}$ rotation axis are shown in green and blue, respectively (their product, the inversion operator, is not shown). 
energy and the selected geometrical parameters just after the TS and reflected in Fig. 7, are likely due to the large energetic difference between reactants and products that makes the process to suddenly accelerate in such a part of the reaction path. The animation of the full set of vibrational modes can be seen in Reference 42 .

Table II reports the energy of the stationary species involved in the recombination path. Both the zero-th point energy (ZPE) and thermal contributions to the Gibbs free energy differences corresponding to set temperatures between $300 \mathrm{~K}$ and $1500 \mathrm{~K}$ were also taken into account by constructing the proper vibrational canonical partition function (for further details see Refs. 43, 44). The electron-nuclear contribution to the activation energy barrier for the V-I recombination has been estimated in $0.81 \mathrm{eV}$. ZPE and thermal contributions reduce this value by about $11 \%$. The activation barrier is nearly constant within the considered temperature range. These results confirm the experimental evidence that full recombination is extremely difficult upon ion implantation followed by high-temperature annealing. [45]

The structure of the TS is schematically reported in Figure 6. In comparison with the $\mathrm{VI}_{1}$ structure shown in Figure 5, it results that the couple of symmetry equivalent atoms labeled as A and A' evolves by rotating around the $C_{2}$ symmetry axis. Concurrently with such a rotation, both atoms decrease their coordination from four to three acquiring a planar configuration with bond angles close to $120^{\circ}$. These features, together with the slight reduction of the A-A' bond length in the TS, reveal a change of the valence orbitals hybridization from $\mathrm{sp}^{3}$ to $\mathrm{sp}^{2}$ with a weak $\pi$ bonding character between such atoms. In the TS, the distance of the originally bonded $\mathrm{A}$ and $\mathrm{E}$ atoms increases up to $2.09 \AA$, the latter keeping the $\mathrm{sp}^{3}$ hybridization as suggested by the pyramidal coordination with its three neighboring atoms.

To get more insight into the electronic structure changes along the process, let us consider the charges of the mainly involved atoms, as documented in the left panel of Figures 5 and 6 . They have been obtained through the periodic extension of the HirshfeldI procedure.[41] It turns out that the atoms that migrate from the interstitial to the perfect diamond positions (A, A') display a slight electron deficiency, while their first neighbors (D, E) display some electron excess. This is fully consistent with their respective carbeniumand carbanion-like nature. Accordingly, the recombination resembles a kind of concerted $S_{N} 1$-type reaction [46] similar to those well characterized in organic chemistry.

The nucleophiles are in this case the carbanions around the defect (atom $\mathrm{E}$ and its symmetry-equivalent one). According to this, carbenium-like ions feature a charge of $0.09|e|$ which is practically a half in absolute value of that displayed by carbanion-like ones $(-0.18|e|)$. This might be attributed to the fact that in the former case part of the electrons are involved in a weak $\pi$-bonding, as mentioned above. The charges on the remaining atoms are one order of magnitude smaller than the previous
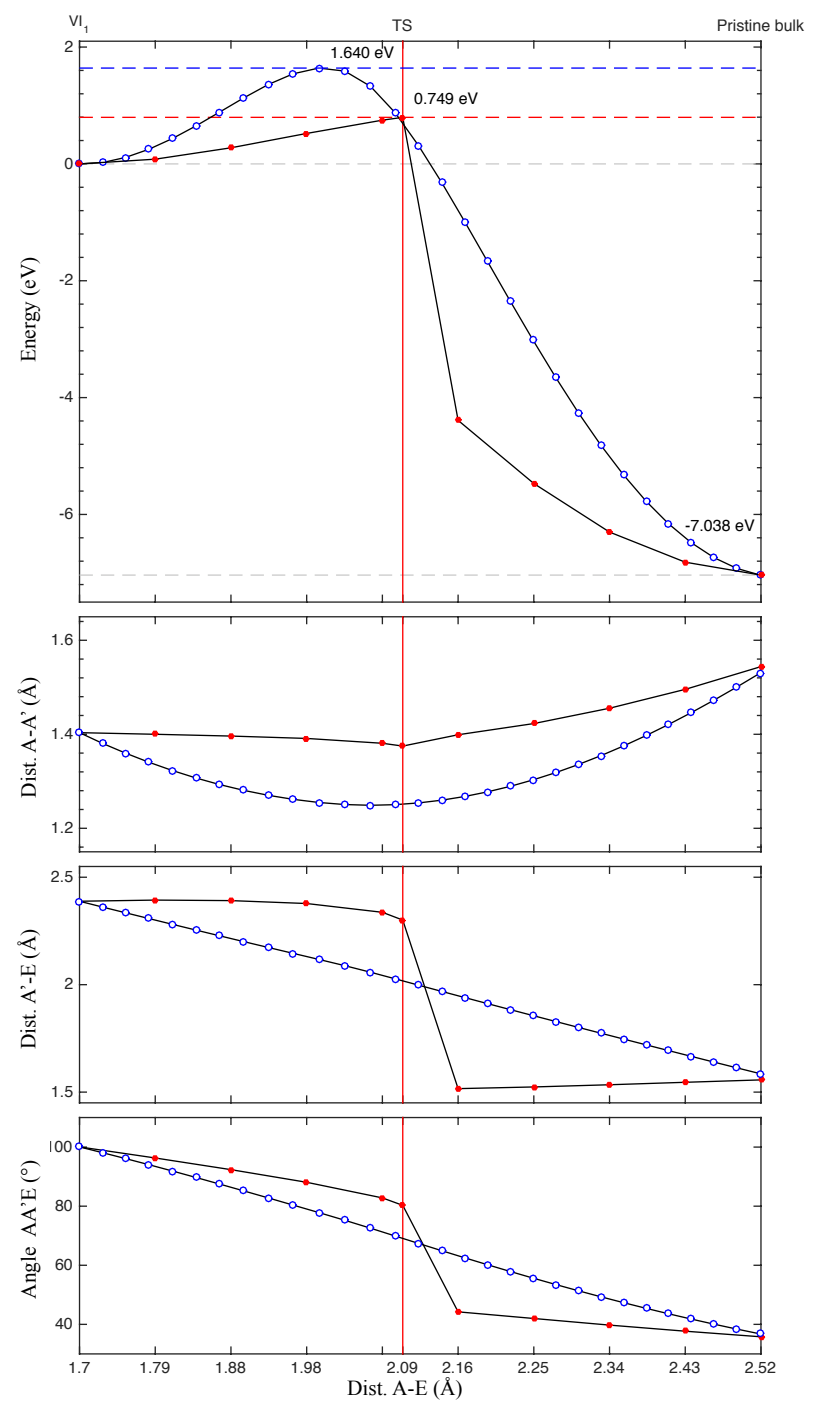

FIG. 7: (color online) Activation barrier for the recombination of the $\mathrm{VI}_{1}$ double defect (left) to give pristine diamond (right). The full red points refer to the search for the transition state (TS) according to the DRC algorithm; the geometry of $\mathrm{VI}_{1}$ and TS are shown in Figure 5 and Figure 6. Animations of the vibrational modes (including the one along the reaction coordinate) are shown in Ref. 42 (click on "VI1 Freq" and "VI1 TS Freq"). The open blue points have been obtained with a simple model according to which each atom in the unit cell (as well as the lattice parameters) evolve linearly along the path between $\mathrm{VI}_{1}$ and perfect diamond. All calculations have been performed on supercell $\mathrm{S}_{64}$.

ones confirming their close to neutral nature. The slight ionicity of the TS suggests that the process of defect recombination occurring during ion implantation (i.e. "self annealing")[45] is to decrease the energy barrier by creating electrostatic fields which stabilize charge polarization. Accordingly, the kinetics of the process becomes faster allowing defect elmination.

In Figure 7 a second energy profile is shown, which 
TABLE II: Electronic and Gibbs free energy (in hartree) of the starting $\left(\mathrm{VI}_{1}\right)$, transition state (TS) and final (perfect diamond) points of the recombination reaction path. $\Delta \mathrm{E}_{1}$ and $\Delta \mathrm{E}_{2}$ (in eV) are the energy differences TS minus $\mathrm{VI}_{1}$ and TS minus perfect diamond, respectively. Data refer to five different temperature (in $\mathrm{K}$ ) and are obtained with the B3LYP/6-21 method.

\begin{tabular}{ccccccc}
\hline \hline & $\mathrm{T}$ & $\mathrm{VI}_{1}$ & $\mathrm{TS}$ & Pure & $\Delta \mathrm{E}_{1}$ & $\Delta \mathrm{E}_{2}$ \\
\hline $\mathrm{E}_{L}$ & - & -2435.3193 & -2435.2897 & -2435.5789 & 0.81 & 7.87 \\
$\mathrm{E}_{L}+\mathrm{ZPE}$ & - & -2434.8955 & -2434.8693 & -2435.1495 & 0.71 & 7.62 \\
& 300 & -2434.8989 & -2434.8728 & -2435.1526 & 0.71 & 7.61 \\
Gibbs & 600 & -2434.9404 & -2434.9143 & -2435.1924 & 0.71 & 7.57 \\
& 900 & -2435.0377 & -2435.0116 & -2435.2874 & 0.71 & 7.50 \\
& 1200 & -2435.1837 & -2435.1573 & -2435.4308 & 0.72 & 7.44 \\
& 1500 & -2435.3697 & -2435.3428 & -2435.6140 & 0.73 & 7.38 \\
\hline \hline
\end{tabular}

was obtained with a much simpler model, according to which one can imagine that all the inner coordinates and also the lattice parameters linearly evolve from the $\mathrm{VI}_{1}$ geometry to the final one (perfect diamond). About 30 points have been computed along this path. The resulting energy barrier is, as expected, more than twice higher than the one obtained with the more sophisticated DRC strategy.

The above conclusions concerning the nature of the TS and the height of the energy barrier for the recombination of the two defects can obviously change to a certain extent when a different functional, a larger basis set or a larger supercell are adopted. As regards the first point, Table III shows that the barrier varies from $0.81 \mathrm{eV}$ to $1.01 \mathrm{eV}$ for the three hybrid functionals; it drops to 0.62 $\mathrm{eV}$ and $0.67 \mathrm{eV}$ for PBE and LDA, while it increases to $1.62 \mathrm{eV}$ for HF. The effect of the basis set (four sets of increasing size are used with B3LYP, varying from 6-21G to $6-31 \mathrm{G}^{*}$ ) is of the order of $10 \%$ (from $0.72 \mathrm{eV}$ to 0.81 $\mathrm{eV})$; the effect of the supercell, not documented here, is negligible. The TS geometry is about the same for the

TABLE III: Total energy (in hartree) of the starting ( $\left.\mathrm{VI}_{1}\right)$, transition state (TS) and final (perfect diamond) points of the recombination reaction path obtained with various functionals and basis sets. $\Delta \mathrm{E}_{1}$ and $\Delta \mathrm{E}_{2}$ (in eV) are the energy differences TS minus $\mathrm{VI}_{1}$ and TS minus perfect diamond, respectively; in all cases the TS geometry and energy have been determined according to the DRC algorithm. $\delta R$ is the energy gain in the search of the TS geometry when starting from the B3LYP/6-21G TS geometry; it represents then a measure of the difference in the transition state geometries for the various functionals. Only in the LDA case it is a large fraction of $\Delta \mathrm{E}_{1}$.

\begin{tabular}{lc|cccccc}
\hline \hline Functional & Basis Set & $\mathrm{VI}_{1}$ & $\mathrm{TS}$ & Diamond & $\Delta \mathrm{E}_{1}$ & $\delta R$ & $\Delta \mathrm{E}_{2}$ \\
\hline HF & $6-21$ & -2421.4643 & -2421.4049 & -2421.7603 & 1.62 & 0.26 & 9.67 \\
PBE0 & $6-21$ & -2434.5429 & -2434.5058 & -2434.8059 & 1.01 & 0.21 & 8.17 \\
& $6-21$ & -2435.3193 & -2435.2897 & -2435.5781 & 0.81 & - & 7.85 \\
B3LYP & $6-21^{*}$ & -2435.9710 & -2435.9431 & -2436.2229 & 0.76 & 0.01 & 7.61 \\
& $6-31$ & -2436.7811 & -2436.7546 & -2437.0444 & 0.72 & 0.04 & 7.90 \\
& $6-31^{*}$ & -2437.3780 & -2437.3509 & -2437.6340 & 0.74 & 0.15 & 7.70 \\
HSE06 & $6-21$ & -2434.4984 & -2434.4639 & -2434.7613 & 0.94 & 0.20 & 8.09 \\
PBE & $6-21$ & -2434.2414 & -2434.2186 & -2434.4872 & 0.62 & 0.01 & 7.31 \\
LDA & $6-21$ & -2415.8914 & -2415.8667 & -2416.1450 & 0.67 & 0.55 & 7.57 \\
\hline \hline
\end{tabular}

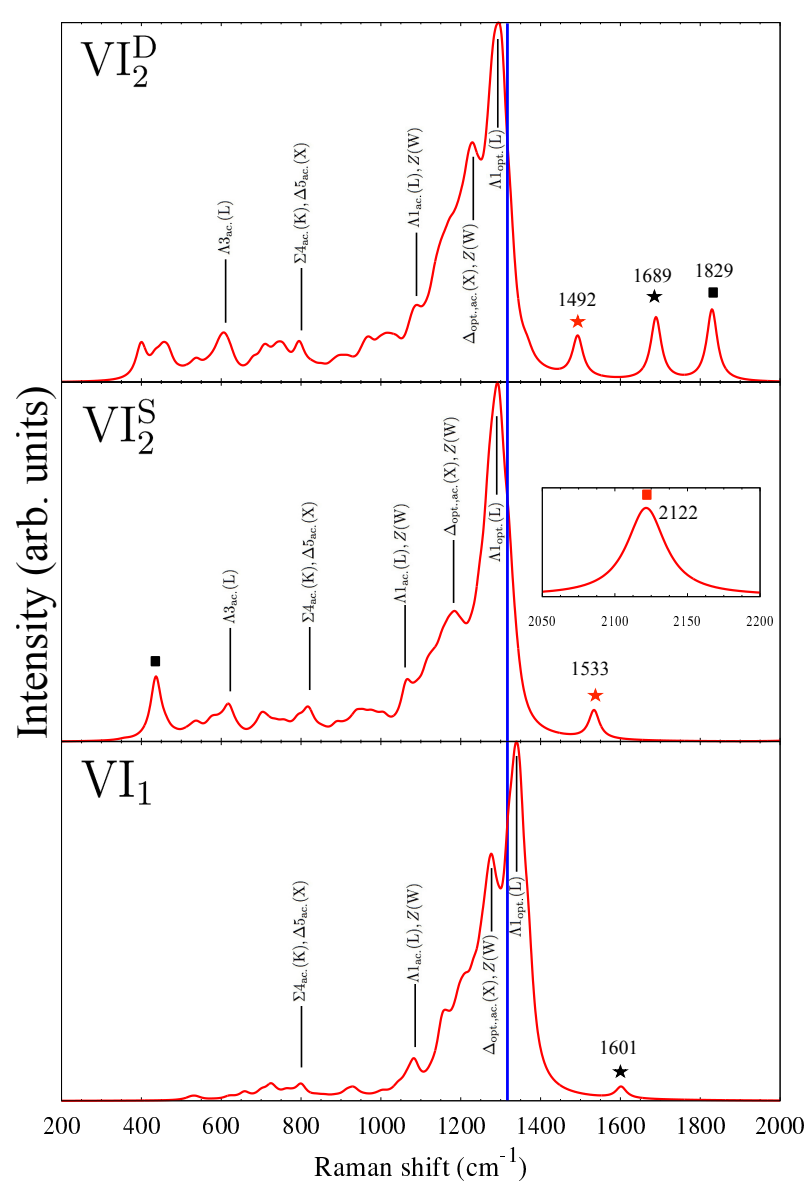

FIG. 8: (color online) Raman spectra of the three different defects at the same concentration $\left(S_{64}\right)$. Red starred and squared dots mark the peaks whose intensities have been multiplied by a factor 12 . Vertical blue line marks the position of the pristine diamond isolated peak (at $1317.0 \mathrm{~cm}^{-1}$ ). The three peaks in the high-frequency range that find a clear correspondence with experimental data are marked with starred dots, while the peaks characterized by a tentative or unclear attribution are marked with square dots. In the low-frequency range, the characteristic vibrational modes of diamond at different symmetry points are labeled.

various functionals, as indirectly documented by $\delta R$, the only exception being LDA.

\section{Vibrational properties}

The Raman and IR spectra can offer a significant contribution to the identification of the different types of defects under consideration. Raman spectra were obtained as described in Section II.A; the same scheme was adopted in previous works $[31,33]$. The position of the first-order Raman line (marked with a continuous vertical blue line in Figure 8) obtained with the present functional and basis set shows a $\sim 15 \mathrm{~cm}^{-1}$ offset with respect to experimental values[49]. 


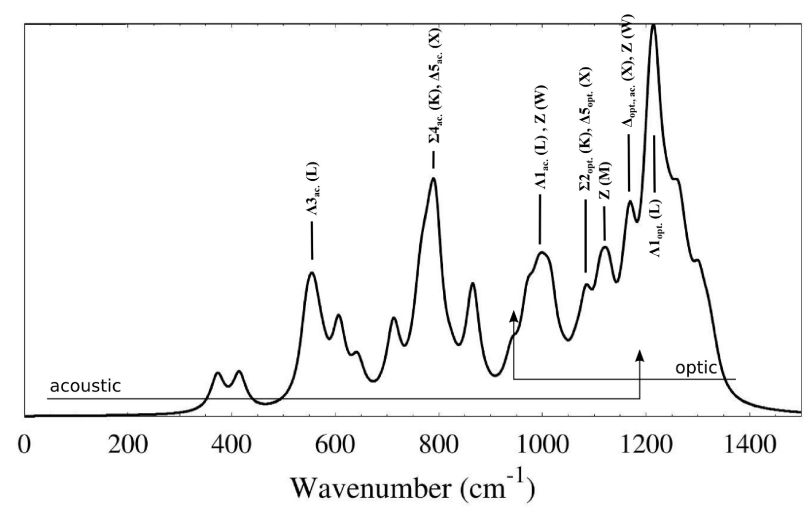

FIG. 9: $A b$ initio simulation of the vibrational density of states of diamond $\left(\mathrm{FWHM}=32 \mathrm{~cm}^{-1}\right)$. Acoustic and optic modes are highlighted at frequencies $<1200 \mathrm{~cm}^{-1}$ and $>1000$ $\mathrm{cm}^{-1}$, respectively[47]. The characteristic vibrational modes of diamond at different symmetry points[48] are labeled.

Figure 8 shows the Raman spectra of the $\mathrm{VI}_{2}^{\mathrm{D}}, \mathrm{VI}_{2}^{\mathrm{S}}$ and $\mathrm{VI}_{1}$ defects. While a general feature associated to all of the reported spectra consists of a large number of Ramanactive modes at low frequencies (i.e. in the 400-1300 $\mathrm{cm}^{-1}$ range), in the high-frequency range (i.e. $>1300$ $\mathrm{cm}^{-1}$ ), few well-defined Raman features appear in the computed spectra. These latter features are extremely significant in the interpretation of the vibrational properties of the defects under investigation, because their frequencies lay in a range where no vibrational modes are present for the pristine diamond structure[47], as confirmed by the vibrational density of states computed in the present work (see Figure 9, in which no vibrational features are observed at $>1300 \mathrm{~cm}^{-1}$ ). The animation in a customizable 3D representation of the defect geometry of all vibrational modes (and in particular the ones which will be discussed in the following) are visible at the web-site reported at Reference 42 .

It was reported in previous simulations that the $\langle 100\rangle$ self split-interstitial is associated to two Raman peaks at $1504 \mathrm{~cm}^{-1}$ and $1897 \mathrm{~cm}^{-1}$ for the same defect concentration reported here, i.e. corresponding to a $\mathrm{S}_{64}$ cell[33]. In the limit at infinite dilution, these peaks respectively shift to $1550 \mathrm{~cm}^{-1}$ and $1927 \mathrm{~cm}^{-1}$, only the former one being also IR active. Conversely, no Raman features in the high-frequency range were observed for the vacancy defect[31]. These results confirmed that the "high frequency" modes cannot be attributed to vibrations of purely-sp ${ }^{3}$ defective phases.

As commonly observed in experimental measurements, several Raman peaks appear in the high-frequency range for defective diamond samples, among which the most prominent ones are centered approximately at $\sim 1450$ $\mathrm{cm}^{-1}, \sim 1490 \mathrm{~cm}^{-1}, \sim 1600 \mathrm{~cm}^{-1}$ and $\sim 1680 \mathrm{~cm}^{-1}$ shifts[50-52]. The peaks at $1492 \mathrm{~cm}^{-1}, 1601 \mathrm{~cm}^{-1}$ and $1689 \mathrm{~cm}^{-1}$ in the computed Raman spectra of $\mathrm{VI}_{2}^{\mathrm{D}}$ and $\mathrm{VI}_{1}$ find a close correspondence with three (out of four) of the above-mentioned widely observed features. Peaks are marked with starred dots in Figure 8; the $1492 \mathrm{~cm}^{-1}$ peak is marked in red (instead of black), since it has been enhanced by a factor 12 to make it more visible. The 1601 $\mathrm{cm}^{-1}$ feature corresponds to a peak which is very strong in defective diamond, and has already been attributed to the self-interstitial defect $[50,53]$. Interestingly, this feature was not observed in a previous simulation by some of the present authors, referring to the Raman modes of the isolated interstitial defect[33], so that we can conclude that its generally accepted attribution is correct only if this defect is first-neighboring a vacancy.

The spectrum of the $\mathrm{VI}_{2}^{\mathrm{D}}$ defect presents a peak at 1829 $\mathrm{cm}^{-1}$ (labeled with a square dot in Figure 8). This feature can possibly find a correspondence with a $25 \mathrm{~cm}^{-1}$ broad Raman peak at $\sim 1815 \mathrm{~cm}^{-1}$ which is experimentally observed in implantated diamonds of different origin and types[48]. Since it has been observed in defective diamonds with different types of defects and impurities, this feature has been tentatively attributed in previous studies to an intrinsic defect of unknown type[48]. From a visual inspection of the corresponding vibrational mode[42], this feature finds an unequivocal (and previously unreported) attribution to the stretching mode of the two interstitial atoms along their bond axis.

It is worth noting that two very weak Raman features appear at $\sim 1533 \mathrm{~cm}^{-1}$ and $\sim 2122 \mathrm{~cm}^{-1}$ (marked with red dots, as multiplied by a factor 12) in the Raman spectrum of defect $\mathrm{VI}_{2}^{\mathrm{S}}$ : the former feature (marked with a red starred dot in the figure) is reported as observed in Reference 50; for the latter (marked with a red square dot) we did not find any mention in experimental papers, possibly due to the low intensity and location in a part of the spectrum frequently not explored.

Finally, in our results there is no indication of a Raman peak at $1450 \mathrm{~cm}^{-1}$ for any of the defects under consideration. Therefore, on the basis of the present results the attribution of the $1450 \mathrm{~cm}^{-1}$ Raman mode found in several experimental works is still unclear.

As for the low-frequency (i.e. $400-1300 \mathrm{~cm}^{-1}$ ) modes, the picture is more articulated and less specific to the defects under investigation. A broad band with many Raman features is widely observed in experimental spectra from defective diamond, which is generally attributed to non-graphitic $\mathrm{sp}^{2}$ defects[50]. They qualitatively resemble the vibrational density of states of pristine diamond, with the vibrations at $>1000 \mathrm{~cm}^{-1}$ being associated to optical modes and the vibrations at $<1200 \mathrm{~cm}^{-1}$ to acoustic modes (see Figure 9)[47]. In general, the presence of the low-frequency modes in defective diamond has been previously interpreted as due to the defect-induced lifting of the symmetry rules which make them Ramaninactive in the pristine crystal structure. Similarly to what observed for the interstitial[33], the Raman spectrum of the $\mathrm{VI}_{1}$ defect exhibits a clear predominance of the optical modes with respect to the acoustic ones, while the Raman spectrum of the $\mathrm{VI}_{2}^{\mathrm{D}}$ defect, and more so the spectrum of the $\mathrm{VI}_{2}^{\mathrm{S}}$ defect, display a stronger contri- 
bution from the low-frequency acoustic modes, in closer resemblance to what observed for the vacancy[31].

The characteristic vibrational modes of diamond (i.e. $\Delta, \Sigma$ and $\Lambda$ modes at different symmetry points, labeled in Figures 8 and 9) find a satisfactory correspondence in our data. As the only possible exception to this interpretation, it is worth noting that the spectra relevant to the $\mathrm{VI}_{2}^{\mathrm{D}}$ and $\mathrm{VI}_{2}^{\mathrm{S}}$ defects display Raman features in the $400-500 \mathrm{~cm}^{-1}$ range (labeled with square dots in Figure 8) which do not find a clear correspondence with the results of experimental measurements. Their interpretation from the visual inspection of the corresponding vibrational modes is not unequivocal[42], and therefore no further considerations can be made on these features.

The simulation of the IR absorption features yielded interesting results which nonetheless can be largely assimilated to those obtained from Raman spectra. Therefore, they are reported and commented in the SM.

\section{CONCLUSIONS}

An extensive simulation of different configurations of the "vacancy + $\langle 100\rangle$ self-interstitial" defect has been performed here, by adopting an ab initio supercell scheme, a local gaussian-type basis set and various "hybrid" functionals, that contain a fraction of exact exchange, in conjuction with the CRYSTAL14 code[34].

A detailed analysis of the electronic states has been performed at first, by comparing the relative stability of the different spin states, and by analyzing their band structure and charge and spin distribution. It turns out that the vicinal configuration $\mathrm{VI}_{1}$ of the defect is significantly more stable than the $\mathrm{VI}_{2}^{\mathrm{D}}, \mathrm{VI}_{2}^{\mathrm{S}}, \ldots, \mathrm{VI}_{\infty}$ configurations, because in this case all the involved atoms can reach a four-fold coordination, despite distances and angles that are quite far from those of pristine diamond. The ground state of the $\mathrm{VI}_{1}$ defect is a closed shell and its band structure is very similar to the one of pristine diamond: in particular, the gap is free from defect states.

The mechanisms underlying the recombination of the vacancy and interstitial defects have been investigated in detail, thus addressing an open issue which has important implications in the defect engineering of diamond (doping, colour centres creation). The recombination path was explored by using the Distinguished Reaction Coordinate strategy; a barrier ranging from $0.75 \mathrm{eV}$ to 1.01 $\mathrm{eV}$ was determined when using various hybrid functionals; pure DFT (LDA and PBE) produces much lower barriers.
Previous estimates of the recombination barrier in the $1.3 \mathrm{eV}$ and $1.68 \mathrm{eV}[8,20,23]$ interval were obtained with rather indirect approaches, i.e. with phenomenological models applied to the evolution of vacancy-related luminescence and EPR in MeV-electron-implanted diamond samples. The obtained recombination barrier can be compared to the proposed $>4.3 \mathrm{eV}$ barrier for the formation of the stable divacancy defect, as obtained from $a b$ initio DFT modeling[26]. This implies that the formation of divacancies does not seem to represent an effective mechanism competing with or supporting the vacancy/interstitial recombination. While a comprehensive investigation of all possible vacancy/interstitial recombination pathways goes beyond the scope of the present work, in light of these results it seems reasonable to hypothesize the existence of more complex (and yet to be identified) mechanisms accounting for the partial recombination of Frenkel pairs in lightly damaged diamonds, which is experimentally observed upon thermal annealing. Overall, the present results shed light on the understanding of widely observed radiation-damage effects in diamond, and in particular explain why the full recovery of its pristine electro-optical properties to this date still represents such a hard technological challenge.

A detailed analysis of the Raman spectra of the $\mathrm{VI}_{2}^{\mathrm{D}}$, $\mathrm{VI}_{2}^{\mathrm{S}}$ and $\mathrm{VI}_{1}$ defects allowed to unequivocally identify several spectral features at frequencies above the firstorder Raman peak of pristine diamond (most notably, the $\sim 1490 \mathrm{~cm}^{-1}, \sim 1600 \mathrm{~cm}^{-1}$ and $\sim 1680 \mathrm{~cm}^{-1}$ lines), which have been extensively observed in previous experimental studies[50-52]. Moreover, the observation of a $\sim 1829 \mathrm{~cm}^{-1}$ Raman peak from our simulations allowed for a tentative attribution of a previously observed (and unattributed) Raman feature to the stretching of the two interstitial atoms of the $\mathrm{VI}_{2}^{\mathrm{D}}$ defect along their bond axis.

\section{SUPPLEMENTARY MATERIAL}

Detailed description of the computational set up, Mulliken population analysis and simulated IR spectra of the three studied defects.

\section{Acknowledgements}

CMZW thanks Mexican CONACyT for finantial support through project $178853-\mathrm{F}$.
[1] R. B. Jackman. Special Issue: Diamond ElectronicsPreface. Semicond Sci Tech, 18(3):1-2, 2003.

[2] R. J. Nemanich, J. A. Carlisle, A. Hirata, and K. Haenen. CVD Diamond - Research, Applications, and Challenges. MRS Bulletin, 39(06):490-494, 2014.
[3] R. Job, M. Werner, A. Denisenko, A. Zaitsev, and W. R. Fahrner. Electrical Properties of Lithium Implanted Layers on Synthetic Diamond. Diam. Relat. Mater., 5:757760, 1996.

[4] C. Uzan-Saguy, R. Kalish, R. Walker, D. N. Jamieson, 
and S. Prawer. Formation of Delta-Doped, Buried Conducting Layers in Diamond by High-Energy B-Ion Implantation. Diam. Relat. Mater., 7:1429-1432, 1998.

[5] F. C. Waldermann, P. Olivero, J. Nunn, K. Surmacz, Z. Y. Wang, D. Jaksch, R. A. Taylor, I. A. Walmsley, M.Draganski, P. Reichart, A. D. Greentree, D. N. Jamieson, and S. Prawer. Creating Diamond Color Centers for Quantum Optical Applications. Diam. Relat. Mater., 16:1887-1895, 2007.

[6] S. Pezzagna, D. Rogalla, D. Wildanger, J. Meijer, and A. Zaitsev. Creation and Nature of Optical Centres in Diamond for Single-Photon Emission - Overview and Critical Remarks. New J. Phys., 13:035024, 2011.

[7] G. Davies, S. C. Lawson, A. T. Collins, A. Mainwood, and S. J. Sharp. Vacancy-related centers in diamond. Phys. Rev. B, 46:13157-13170, 1992.

[8] L. Allers, A. T. Collins, and J. Hiscock. The Annealing of Interstitial-Related Optical Centres in Type II Natural and CVD Diamond. Diam. Relat. Mater., 7:228-232, 1998.

[9] G. Davies. Charge States of the Vacancy in Diamond. Nature, 269:498-500, 1977

[10] N. Tsubouchi, M. Ogura, N. Mizuochi, and H. Watanabe. Electrical Properties of a B Doped Layer in Diamond Formed by Hot B Implantation and High-Temperature Annealing. Diam. Relat. Mater., 18:128-131, 2009.

[11] J. Schwartz, P. Michaelides, C. D. Weis, and T. Schenkel. In situ optimization of co-implantation and substrate temperature conditions for nitrogen-vacancy center formation in single-crystal diamonds. New J. Phys., 13:035022, 2011.

[12] B. Naydenov, V. Richter, J. Beck, M. Steiner, P. Neumann, G. Balasubramanian, J. Achard, F. Jelezko, J. Wrachtrup, and R. Kalish. Enhanced Generation of Single Optically Active Spins in Diamond by Ion Implantation. Appl. Phys. Lett., 96:163108, 2010.

[13] B. Naydenov, F. Reinhard, A. Laemmle, V. Richter, R. Kalish, U. F. S. D'Haenens-Johansson, M. Newton, F. Jelezko, and J. J. Wrachtrup. Increasing the Coherence Time of Single Electron Spins in Diamond by High Temperature Annealing. Appl. Phys. Lett., $97(24): 242511,2010$.

[14] D. Gatto Monticone, F. Quercioli, R. Mercatelli, S. Soria, S. Borini, T. Poli, M. Vannoni, E. Vittone, and P. Olivero. Systematic Study of Defect-Related Quenching of NV Luminescence in Diamond with Time-Correlated Single-Photon Counting Spectroscopy. Phys. Rev. B, 88:155201, 2013.

[15] J. Forneris, V. Grilj, M. Jaksic, A. Lo Giudice, P. Olivero, F. Picollo, N. Skukan, C. Verona, G. Verona-Rinati, and E. E. Vittone. IBIC Characterization of an IonBeam-Micromachined Multi-Electrode Diamond Detector. Nucl. Instrum. Meth. B, 306:181-185, 2013.

[16] F. Picollo, D. Gatto Monticone, P. Olivero, B. A. Fairchild, S. Rubanov, S. Prawer, and E. Vittone. Fabrication and Electrical Characterization of ThreeDimensional Graphitic Microchannels in Single Crystal Diamond. New J. Phys., 14:053011, 2012.

[17] N. Tsubouchi, H. Umezawa, Y. Mokuno, A. Chayahara, and S. Shikata. Lattice Structure of a Freestanding Nitrogen Doped Large Single Crystal Diamond Plate Fabricated Using the Lift-Off Process: X-Ray Diffraction Studies. Diam. Relat. Mater., 25:119-123, 2012.

[18] R. Kalish. The Role of Ion-Implantation in the Realiza- tion of Spintronic Devices in Diamond. Nucl. Instrum. Meth. B, 272:42-48, 2012.

[19] J. F. Prins. Point-Defect Interactions when Annealing Diamonds Implanted at Low Temperatures. Phys. Rev. $B, 44(6): 2470-2479,1991$.

[20] M. E. Newton, B. A. Campbell, D. J. Twitchen, J. M. Baker, and T. R. Anthony. RecombinationEnhanced Diffusion of Self-Interstitial Atoms and Vacancy-Interstitial Recombination in Diamond. Diam. Relat. Mater., 11:618-622, 2002.

[21] J. F. Prins. On the Annihilation of Vacancies by Diffusing Interstitial Atoms in Diamond. Diam. Relat. Mater., 9:1853-1839, 2000.

[22] J. F. Prins and T. E. Derry. Radiation Defects and their Annealing Behaviour in Ion-Implanted Diamonds. Nucl. Instrum. Meth. B, 166-167:364-373, 2000.

[23] D. J. Twitchen, D. C. Hunt, C. Wade, M. E. Newton, J. M. Baker, T. R. Anthony, and W. F. Banholzer. The Production and Annealing Stages of the Self-interstitial (R2) Defect in Diamond. Physica B, 273-274:644-646, 1999.

[24] K. Iakoubovskii and G. J. Adriaenssens. Trapping of Dacancies by Defects in Diamond. Journal of Physics: Condensed Matters, 13(26):6015, 2001.

[25] J. F. Prins. Vacancy diffusion and trapping in electronirradiated type IaA diamonds. Diam. Relat. Mater., 10:87-93, 2001.

[26] B. Slepetz and M. Kertesz. Divacancies in Diamond: a Stepwise Formation Mechanism. Phys. Chem. Chem. Phys., 16(4):1515-1521, 2014.

[27] C. A. Coulson and Mary J. Kearsley. Colour Centres in Irradiated Diamonds. I. Proc. R. Soc. A, 241(1227):433454, 1957.

[28] S. J. Breuer and P.R. Briddon. Ab Initio Investigation of the Native Defects in Diamond and Self-Diffusion. Phys. Rev. B, 51(11):6984-6994, 1995.

[29] J. P. Goss, B. J. Coomer, R. Jones, T. D. Shaw, P. R. Briddon, M. Rayson, and S. Öberg. Self-Interstitial Aggregation in Diamond. Phys. Rev. B, 63(19):195208, 2001.

[30] D. Hyde-Volpe, B. Slepetz, and M. Kertesz. The $[\mathrm{V}-\mathrm{C}=\mathrm{C}-\mathrm{V}]$ Divacancy and the Interstitial Defect in Diamond: Vibrational Properties. J. Phys. Chem., 114(21):9563-9567, 2010.

[31] J. Baima, A. Zelferino, P. Olivero, A. Erba, and R. Dovesi. Raman spectroscopic features of the neutral vacancy in diamond from ab initio quantum-mechanical calculations. Physical Chemistry Chemical Physics, 18:1961-1968, 2016.

[32] A. Zelferino, S. Salustro, J. Baima, V. Lacivita, R. Orlando, and R. Dovesi. The electronic states of the neutral vacancy in diamond: a quantum mechanical approach. Theor. Chem. Acc., 135(3):1-11, 2016.

[33] S. Salustro, A. Erba, C. M. Zicovich-Wilson, Y. Nöel, L. Maschio, and R. Dovesi. Infrared and Raman Spectroscopic Features of the Self-Interstitial Defect in Diamond from Exact-Exchange Hybrid DFT Calculations. Phys. Chem. Chem. Phys., 2016.

[34] R. Dovesi, R. Orlando, A. Erba, C. M. Zicovich-Wilson, B. Civalleri, S. Casassa, L. Maschio, M. Ferrabone, M. De La Pierre, P. D'Arco, Y. Noël, M. Causà, M. Rérat, and B. Kirtman. CRYSTAL14: A Program for the $A b$ Initio Investigation of Crystalline Solids. Int. J. Quan- 
tum Chem., 114:1287, 2014.

[35] R. Dovesi, V. R. Saunders, C. Roetti, R. Orlando, C. M. Zicovich-Wilson, F. Pascale, B. Civalleri, K. Doll, N. M. Harrison, I. J. Bush, Ph. D'Arco, and M. Llunell. CRYSTAL 2014 User's Manual. . University of Torino, Torino, 2013.

[36] M. J. Rothman, J. L. Lohr, C. S. Ewig, and J. R. V. Wazer. Application of the energy minimization method to a search for the Transition State for the $\mathrm{H}_{2}+\mathrm{D}_{2}$ exchange reaction. In D. G. Truhlar, editor, Potential Energy Surfaces and Dynamical Calculations, pages 653660. Plenum, New York, 1979.

[37] A. Rimola, C. M. Zicovich-Wilson, R. Dovesi, and P. Ugliengo. Search and Characterization of Transition State Structures in Crystalline Systems Using Valence Coordinates. J. Chem. Theory Comput., 6:1341-1350, 2010.

[38] P. Pulay and G. Fogarasi. Geometry Optimization in Redundant Internal Coordinates. J. Chem. Phys., 96(4):2856-2860, 1992.

[39] C. Peng, P. Y. Ayala, H. B. Schlegel, and M. J. Frisch. Using Redundant Internal Coordinates to Optimize Equilibrium Geometries and Transition States. J. Comput. Chem., 17(1):49-56, 1996.

[40] J. M. Bofill. Updated Hessian Matrix and the Restricted Step Method for Locating Transition Structures. J. Comput. Chem., 15(1):1-11, 1994.

[41] C. M. Zicovich-Wilson, M. Hô, A. M. Navarrete-López, and S. Casassa. Hirshfeld-I Charges in Linear Combination of Atomic Orbitals Periodic Calculations. Theor. Chem. Acc., 135:188, 2016. DOI: 10.1007/s00214-0161942-5.

[42] The $\mathrm{V}+\mathrm{I}$ defects in diamond, and their possible recombination. http://www.pmmp.jussieu.fr/yves/diamond/.

[43] A. Erba, M. Shahrokhi, R. Moradian, and R. Dovesi. On how Differently the Quasi-Harmonic Approximation Works for two Isostructural Crystals: Thermal Properties of Periclase and Lime. J. Chem. Phys., 142(4):044114, 2015.

[44] R. Demichelis, P. Raiteri, J. D. Gale, and R. Dovesi. Examining the Accuracy of Density Functional Theory for
Predicting the Thermodynamics of Water Incorporation into Minerals: the Hydrates of Calcium Carbonate. $J$. Phys. Chem. C, 117(34):17814-17823, 2013.

[45] J. F. Prins. Activation of Boron-Dopant Atomsin IonImplanted Diamonds. Phys. Rev. B, 38(8):5576-5584, 1988.

[46] Francis A. Carey and Richard J. Sundberg. Advanced Organic Chemistry: Part A: Structure and Mechanisms. Springer Science+Business Media, New York, Fifth edition, 2007.

[47] C. Z. Wang and K. M. Ho. Structure, Dynamics, and Electronic Properties of Diamondlike Amorphous Carbon. Phys. Rev. Lett., 71:1184-1187, 1993.

[48] A. M. Zaitsev. Optical Properties of Diamond - A Data Handbook. Springer-Verlag, 2001.

[49] A first-order Raman shift of $1333 \mathrm{~cm}^{-1}$ for perfect diamond was reported by some of the present authors in a previous study [31]. In a more recent paper[33] the peak position was corrected to $1317 \mathrm{~cm}^{-1}$, as reported also in the present study, the difference being due to the non fully converged grid used [31] for the integration of the exchange and correlation functional (only 55 radial points and 434 angular points are adopted, instead of a more denser 75 radial and 974 angular points integration grid). The stability of the vibrational peaks with respect to all other computational parameters has also been checked.

[50] S. Prawer, K. W. Nugent, and D. N. Jamieson. The Raman Spectrum of Amorphous Diamond. Diam. Relat. Mater., 7:106-110, 1998.

[51] R. Kalish, A. Reznik, S. Prawer, D. Saada, and J. Adler. Ion-Implantation-Induced Defects in Diamond and Their Annealing: Experiment and Simulation. Phys. Status Solidi A, 174(1):83-99, 1999.

[52] J. O. Orwa, K. W. Nugent, D. N. Jamieson, and S. Prawer. Raman Investigation of Damage Caused by Deep Ion Implantation in Diamond. Phys. Rev. B, 62(9):5461-5472, 2000.

[53] D. T. Morelli, T. A. Perry, and J. W. Farmer. Phonon Scattering in Lightly Neutron-Irradiated Diamond. Phys. Rev. B, 47(1):131-139, 1993. 